\title{
Central Cemento-Ossifying Fibroma of Mandible Mimicking Residual Cyst.A Treatment Protocol For Small, Asymptomatic Radiolucencies in oral Cavity.
}

\author{
Dr.Vijaylaxmishettar ${ }^{1}$,Dr S M Kotrashetti ${ }^{2}$ \\ ${ }^{\text {I}}$ (Reader, Department Of Oral And Maxillofacial Surgery, KLE VK Institute Of Dental Sciences, KLE University, \\ Belagavi, India) \\ ${ }^{2}$ (Professor, Department Of Oral And Maxillofacial Surgery, KLE VK Institute Of Dental Sciences, KLE University, \\ Belagavi, India)
}

\begin{abstract}
Presenting an unusual case of asymptomatic central cemento-ossifying fibroma in missing first mandibular molar region (measuring about $1 \mathrm{~cm}$ in its maximum dimension) discovered incidentally on routine radiographic examination. Based on the above case and by literature review, treatment protocol for small asymptomatic radiolucencies in oral cavity has been put forward.
\end{abstract}

Keywords: Asymptomatic radiolucency, cemento-ossifying fibroma, missing tooth, residual cyst.

\section{Introduction}

Fibrous dysplasia, ossifying fibroma, cemento-ossifying fibroma(COF), cementifying fibroma are lesions which contain both fibrous and osseous content [1]. The ossifying fibroma originates from bone whereas COF is odontogenic in origin [2]. COF are benign fibro-osseous lesions of the jaw. These are also known as central ossifying fibroma or central cementifying fibroma. COF originates from periodontal ligament [3]. Periodontal membrane which surrounds the roots is a fibrous connective layer containing multipotential cells. These cells are capable of forming cementum, bone or fibrous tissue [1]. COF contains fibrous tissue and varying amounts calcified tissue resembling bone, cementum or both. Radiographically these are well circumscribed, expansile bony lesion with calcified matrices. These commonly occur in mandible but rarely can involve maxilla and other bones ,mayeven present as extra-skeletal mass in masticator and parapharyngeal space [4]. The maxillary tumors may enlarge in size to involve nasal septum, orbital floor, infra-orbital foramen and maxillary sinus [5]. Here we present an unusual case of asymptomatic central cemento-ossifying fibroma in missing first mandibular molar region (measuring about $1 \mathrm{~cm}$ in its maximum dimension) discovered incidentally on routine radiographic examination.

\section{Case report}

A female patient aged 35 years reported to department of oral and maxillofacial surgery, with history of pain in left back region of lower jaw since 1 month. Clinical examination revealed deep caries with left mandibular second premolar tooth and missing left mandibular first molar. History of exfoliation due to mobility of left mandibular first molar one year back was given by the patient. No relevant medical history and extra-oral presentation were noted. Intra-oral periapical radiograph revealed a well circumscribed radiolucent area with smooth sclerotic border measuring less than $1 \mathrm{~cm}$ in its maximum dimension in the periapical area of missing mandibular first molar (figure 1). Radiolucency in relation to left mandibular second premolar was diagnosed as periapical abscess. A provisional diagnosis of residual cyst was given with respect to the radiolucent area present in left mandibular first molar region. Histopathology report following an excisional biopsy suggested of central cementoossifying fibroma of mandible. 


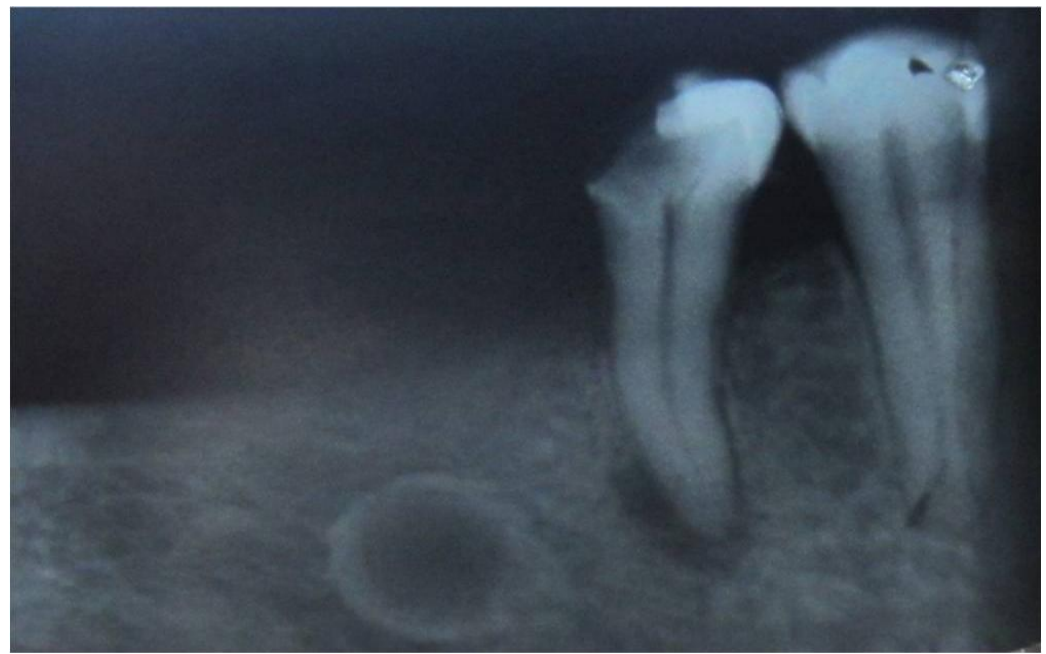

Fig 1.Intra-oralperi-apical radiograph showing a well circumscribed radiolucent area with smooth sclerotic border in the periapical area of missing mandibular first molar and periapical abscess with second premolar

\section{Discussion}

In the year 1872, Menzel gave the first description of cemento-ossifying fibroma, in a 35 year old female who presented with a large tumor in mandible from a long time [2].Traumatic and developmental theories have been put forward for the origin of COF. Nasopharyngeal COF originate from embryologic nests. Differentiation of periodontal membrane from primitive mesenchymal cells may lead to development of COF in different locations like petrous bone, ethmoidal bone etc [4]. Trauma, dental extraction, infection can act as stimulating factors for periodontal membrane to produce cementum [6]. Infection results in inflammation and fibrosis of periapical area which stimulates the remnants of periodontal tissue that is attached to alveolus that lead to origin of COF [4].

In present case patient presented with history of left mandibular first molar exfoliation due to mobility one year back in the same region where the COF had developed.COF commonly occurs in females, between 20 and 40 years of age [3]. In the present case the patient was a 35 year old female.

These commonly occur in mandible, $62 \%$ to $89 \%$ cases occur in mandible, $77 \%$ in premolar region [3]. But rarely can involve maxilla, occiput, ethmoid sinus, sphenoid sinus, temporal bone, orbit, nasal bones, maxillary sinus, nasopharynx and may even rarely present as extra-skeletal mass in masticator and parapharyngeal space [4]. In present case the COF was present in mandible which was asymptomatic, Most of the COF are slow growing and asymptomatic[6]. COF usually are small in size and are discovered on routine dental radiographs. When they enlarge they can lead to swelling, abnormal bite etc.One main reason for large size of maxillary tumors at the time of diagnosis is that large space is available in maxillary sinus into which the COF can expand[3]. The maxillary tumors may enlarge in size to involve nasal septum, orbital floor, infra-orbital foramen and maxillary sinus[5]. In present case the COF was found on the routine examination when patient had come for treatment of other teeth. Central COF are spherical, well circumscribed, solitary radiolucencies with scattered radio-opaque foci. They may cause tooth divergence ${ }^{5}$. Important diagnostic feature of COF is centrifugal growth pattern, as it expands in all directions producing a round tumor mass. The buccal and lingual cortical plates are usually thinned out, but margins remain intact concluding the benign nature of the lesion.

In maxillary COF there is upward bowing of the floor of the maxillary sinus and in mandibular lesion in inferior bowing of the lower border of mandible[6].In present case the intra-oral periapical radiograph revealed a well circumscribed radiolucent area with smooth sclerotic border measuring about $1 \mathrm{~cm}$ in its maximum dimension in the periapical area of missing mandibular first molar.The differential diagnosis in above case include residual cyst[7] and anterior variant of Stafne bone defect due to sublingual salivary gland located in premolar region of mandible [8]. Excision is the recommended treatment for central cemento-ossifying fibroma. In most of the cases the COF especially in mandible shell out easily from the surrounding bone during surgery. Whereas maxillary COF are difficult to remove due to difference in bone character and expansion in maxillary sinus[3]. In the above case the COF was well demarcated from surrounding bone and was excised.

Based on the above case and by literature review, we propose the treatment protocol for small, asymptomatic radiolucencies in oral cavity. 
- Consider various possibilities in differential diagnosis such as artifact, anatomical structures, developmental, pathological causes

- Inform and explain the patient about the asymptomatic radiolucencies

- Imaging modalities CBCT, CT scan can be advised depending on the case.

○ Biopsy followed by treatment has to be carried out

○ Keep patient on follow up

In some cases patients may not agree for biopsyas the radiolucencies are small and asymptomatic. In such cases

- Note it on the case paper :site, size, shape etc of the radiolucency

○ Explain the patient about the possible consequences of not undergoing treatment of such conditions

○ Take a written informed consent

○ Schedule the patient for regular follow up

\section{Conclusion}

In view of limited availability of literature, we want to highlight the rare nature of cemento-ossifying fibroma which resembles residual cyst. This case report also signifies need to take biopsy of asymptomatic radiolucencies in oral cavity and also the treatment protocol is discussed.

\section{References}

[1]. SaritaMagu, Raj Kumar Airon, Daya Shankar Mishra, Rohtas K Yadav, VikasKakkar,Cemento-ossifying fibroma of the maxilla, Indian J Radiol Imaging,10(2), 2000,103-104.

[2]. Hashmi G. Sarwar, M. K. Jindal, SamshadAhmad. A Case Report of Cemento-Ossifying Fibroma,J. Maxillofac Oral Surg , 9(2) , June $2010,178-181$.

[3]. A. John Kuta, C. MacDonald Worley and George E. Kaugars. Central Cemento-ossifying Fibroma of the Maxillary Sinus: A Review of Six Cases. AJNR ,16, Jun 1995,1282-1286.

[4]. So Lyung Jung, Kyu Ho Choi, Young Ha Park, Hyun Chul Song and MiSeon Kwon. Cemento-Ossifying Fibroma Presenting as a Mass of the Parapharyngeal and Masticator Space. AJNR Am J Neuroradiol , October 1999,20,1744-1746.

[5]. Demetrio Tamiolakis, VasiliosThomaidis, IoanisTsamis. Cemento-ossifying Fibroma of the Maxilla: a Case Report. ActaStomat Croat,39(3), 2005,319-321.

[6]. R Mithra, PavitraBaskaran, and M Sathyakumar. Imaging in the Diagnosis of Cemento-Ossifying Fibroma: A Case Series. J Clin Imaging Sci.2, 2012, 52.

[7]. Wen-wei Xia, Ya-qin Zhu, Xiao-yi Wang. Six cases report of differential diagnosis of periapical diseases. Int J Oral Sci,3, 2011, 153159.

[8]. Sisman Y, Etöz OA, Mavili E, Sahman H, TarimErtas E. Anterior Stafne bone defect mimicking a residual cyst: a case report. DentomaxillofacRadiol., 39(2), 2010 Feb,124. 